\title{
Numerical Research on Amplitude of Batoid
}

\author{
Zhijun $\mathrm{Wu}^{*}{ }^{* 1}$, Shengjun $\mathrm{Shi}^{1}$, Weishan $\mathrm{Chen}^{1}$ and Dan $\mathrm{Xia}^{2}$ \\ ${ }^{1}$ School of Mechatronics Engineering, Harbin Institute of Technology, Harbin, Heilongjiang, 150001, China \\ ${ }^{2}$ School of Mechanical Engineering, Southeast University, Nanjing, Jiangsu, 211189, China
}

\begin{abstract}
This paper developed a biomimetic batoid model to investigate the effect of amplitude on propulsion performance. The contour of model's pectoral fins was derived from natural batoid fish, and the model was made to simulate the locomotion of biological fish through user defined functions linked to Fluent. Numerical simulations of model were conducted at five different amplitude indices from 0.08 to 0.32 with increments of 0.06 . The simulation results show that the forward swimming velocity of model increases as amplitude increases. At same time, the excellent propulsion performance emerges at the amplitude index of 0.14 , which is consistent with conventional amplitude of living batoid fish.
\end{abstract}

Keywords: Amplitude, Pectoral fin, Fluent, Numerical simulation.

\section{INTRODUCTION}

To date, bionic underwater vehicles that mimic the swimming mechanisms of natural fish in terms of morphology have been developed to facilitate surveillance for ocean environmental protection and reconnaissance. With the development of advanced smart materials and modern control technologies, vehicles imitating organisms naturally evolved over tens of thousands of years have become a reality. Among the natural underwater creatures, batoid fish have attracted much attention as their large pectoral fins provide high agility [1-3].

Batoid fish propel themselves through the water primarily either with their body and tail (axial-based locomotion) or with their greatly expanded pectoral fins (pectoral-fin-based locomotion) [4]. In the form of pectoral-fin-based locomotion, the pectoral fin is generally actuated synchronously and symmetrically with the other in forward swimming. Pectoralfin-based locomotion has been divided into two categories: oscillating and undulating. Oscillating of the pectoral fins is more similar to flapping in birds; the fins move up and down with a wavelength of $\lambda / \mathrm{BL}<0.5$ (BL denotes body length of the organism). While undulating of the pectoral fins, termed rajiform locomotion, is defined by having more than one wave present on the fins at a time. The intermediate between half a wave and one wave present on the fins are classified into the above two categories in terms of motion pattern.

Highly maneuverable fish have fins that are very effective at manipulating fluid and producing forces in threedimension [5-7]. The propulsion performance of the pectoral fins is affected by various parameters, including the geometry of the fin, the length and the flexibility of the fins, as well frequency and wavespeed etc [8-10]. According to the observation, burrfish increase swimming speed by increasing their fin beat frequency. Computational fluid simulations and experimental results have obtained similar conclusions in other fishes $[11,12]$. Larval plaice and zebrafish have been shown to alternate their pectoral fins during slow locomotion $[13,14]$. In the present study, we numerically simulate the effects of structural and kinematic parameters upon the performance of a simplified batoid model in rajiform swimming. In particular, we concentrate upon the effects of the amplitude of the motion. For this purpose, a three-dimensional bionic batoid model is firstly developed, then the kinematics of the model need to be solved before the fluid-structure interaction problems are solved, finally the quantitative data are recorded to analyze the effect of pectoral fin amplitude on the hydrodynamic performance.

\section{MATERIALS AND METHODS}

\subsection{Geometrical Model}

Assuming Batoid fish are characterized by the streamlined flat body and enlarged pectoral fins which generate the majority of the thrust. Raja eglanteria, a species of batoid, as shown in Fig. (1), classified into the rajiform locomotion with only 0.9 waves on the pectoral fin at a time due to its pectoral fin movements, are selected as the bionic prototype in this paper.

The central body part of the Raja eglanteria is stiffer than the fins and is seldom deformed during swimming, whereas the two pectoral fins are sufficiently flexible to generate elastic fins motion. The tail of raja eglanteria is not considered when developing the bionic model, on one hand, this paper focuses on the linear forward swimming while tail part generates negligible thrust during forward swimming, on the other hand, to simplify the geometrical model in this study. Thus, the geometrical model is divided into three parts: the 


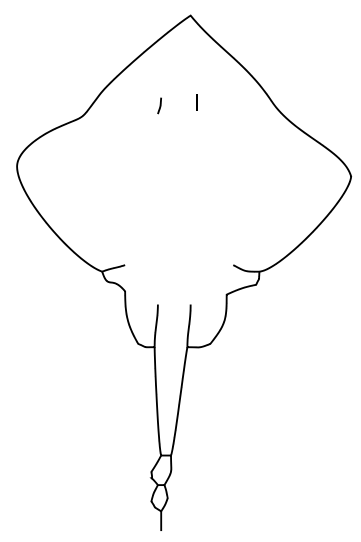

Fig. (1). Planform of Raja Eglanteria.

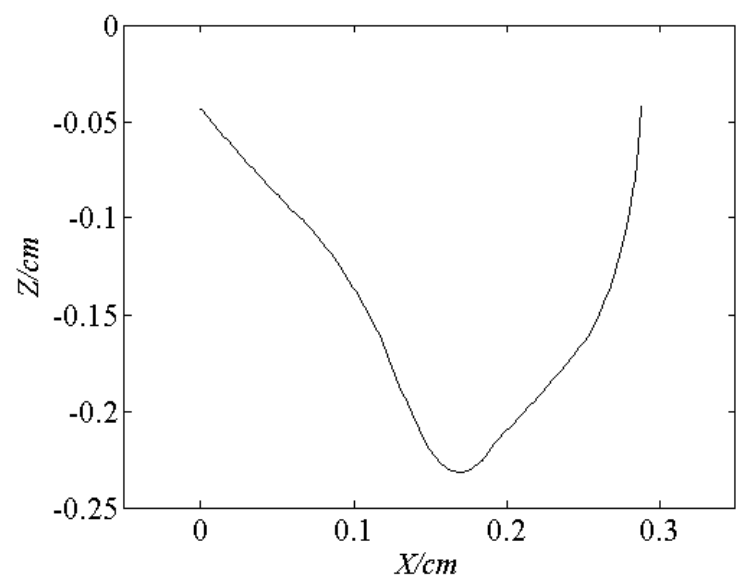

Fig. (2). Contour of Pectoral Fin.

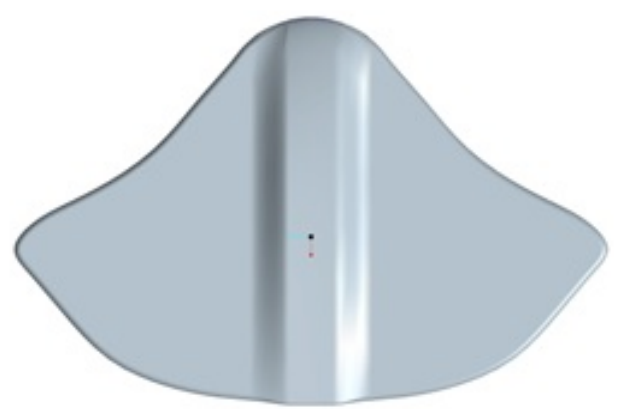

Fig. (3). Physical Model of bionic batoid fish.

body and two pectoral fins. In order to resemble and inherit the low drag characteristics of biological batoid body part, an AG24 aerofoil profile is employed as the sectional shape of the body part of the geometrical model. Two-order piecewise functions are used to approach the contour of biological pectoral fins. The pectoral fin shape with respect to the fin length is shown in Fig. (2).

Since the thickness of the pectoral fin varies slightly, uniform thickness (in y direction) of $1 \mathrm{~cm}$ is specified to the model. The ultimate geometrical model, as shown in Fig. (3), with body length (BL) (in $\mathrm{x}$ direction) and disc width (in $\mathrm{z}$ direction) is of $30.6 \mathrm{~cm}$ and $46.4 \mathrm{~cm}$ respectively.

\subsection{Fin Motion Equations}

Raja eglanteria is classified into the rajiform locomotion due to undulating movements on its pectoral fins. Imitating the motion of biological Raja eglanteria, bionic model propels itself forward by undulating its pectoral fins while keeping body part straight. To achieve linear forward swimming (in $\mathrm{x}$ direction), the motions specified on the pectoral fins are simultaneous and symmetrical. The motion equations of the pectoral fins are defined as:

$y(x, z, t)=A(z) \sin (k x-2 \pi f t)$ 


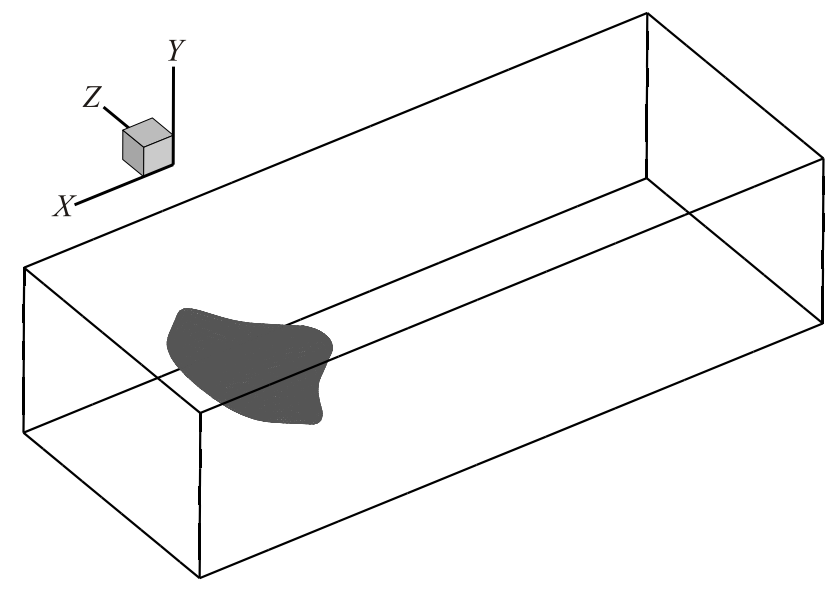

Fig. (4). Sketch of Computational Domain.

Where $A(z)$ is the amplitude controlling equation, $k=2 \pi / \lambda, \lambda$ is the wave length on the fins, $f$ is the oscillating frequency of particles on the fins, $\vec{x}(x, y, z)$ is the threedimensional coordinates values of particles on the geometrical model under body-fitted coordinate system.

The amplitude index $(A I)$ is defined as the ratio of amplitude value to the disc width. To investigate the influence of amplitude on locomotor batoid, $A I$ varies from 0.08 to 0.32 with 0.06 increments in this work. To analyze the effect of flexibility of the pectoral fins on the propulsive performance, two amplitude controlling equations are proposed: linear equation $A_{1}$ and quadratic equation $A_{2}$ :

$$
\begin{aligned}
& A_{1}=A I \times(-0.10696+2.4611 z) \\
& A_{2}=A I \times\left(-0.4628 z+10.727 z^{2}\right)
\end{aligned}
$$

\subsection{Fluids-Structure Interaction}

Since batoid pectoral fins motions require interactions between fluid and structure, the three-dimensional NavierStokes (NS) equations are used to solve the unsteady, incompressible and viscous fluid-structure interactions problems. The density of bionic model is supposed to be the same as that of flow field, thus, gravity of the model is neglected during the calculations. In addition, no slip boundary condition is imposed on the boundary of bionic model. These equations are as follows:

$$
\nabla \cdot \vec{u}=0
$$

$$
\frac{D \vec{u}}{D t}=-\frac{1}{\rho} \nabla p+v \nabla^{2} \vec{u}
$$

$\left.\vec{u}_{c}\right|_{\psi}=\left.\vec{u}_{f}\right|_{\psi}$

where $\vec{u}$ is the velocity of the fluid, $\rho$ and $v$ are the density and kinematic viscosity of flow field respectively, $p$ is the hydrodynamic pressure, $\psi$ is the boundary of fluid-structure interaction, $\vec{u}_{c}$ and $\vec{u}_{f}$ are velocity of deforming boundary of batoid model and fluid velocity at the batoid boundary respectively. The movement of bionic batoid model immersed in fluid follows the formulations:

$$
\begin{aligned}
& m \vec{x}_{c}=\vec{F} \\
& J_{c} \ddot{\theta}=M
\end{aligned}
$$

Where $m$ is gross mass of the model, $\overline{\ddot{x}}_{c}$ is the acceleration of centroid, $\vec{F}$ is the force acting on the model, $J_{c}$ is the inertial moment about the centroid, $\ddot{\theta}$ is the angular acceleration and $M$ is the torque acting on the model.

\subsection{Numerical Method}

In this study, a flow field with $8 \mathrm{~m}$ in length (in $\mathrm{x}$ direction), $4 \mathrm{~m}$ in width (in $\mathrm{z}$ direction) and $1.0 \mathrm{~m}$ in height (in $\mathrm{y}$ direction), is divided into about 5 million tetrahedral grid cells in Gambit (about 6 million tetrahedral grid cells are also carried out, simulation results with less than $3 \%$ of the difference between the coarse grid and the fine grid), a sketch of the computational domain is shown in Fig. (4).

Three-dimensional NS equations are discretized by finite volume method in Fluent. To better simulate the underwater locomotion environment of the natural fish, turbulent model of k-epsilon are used. Dynamic mesh technique is introduced to simulation procedure since the flow field varies with the locomotion of bionic model. The motion of pectoral fins of the model is implemented through user-defined functions (UDF) linked to Fluent. Furthermore, layering and local remeshing methods are utilized to reconstruct new grids. Although a much larger time step can help to accelerate the calculations, a small time step is used to prevent the emergence of negative volume and to guarantee stability of numerical computations. A time step for use in the transient CFD analysis is $\Delta t=0.005 \mathrm{~s}$ in this paper. The averaged central processing unit (CPU) time for calculating a cycle is about 2 to 3 days depending on the grid cells under the use of Intel Xeon CUP $2.27 \mathrm{GHz}$ with $32 \mathrm{~GB}$ random access memory. The oscillating frequency of the pectoral fin is 2.2 


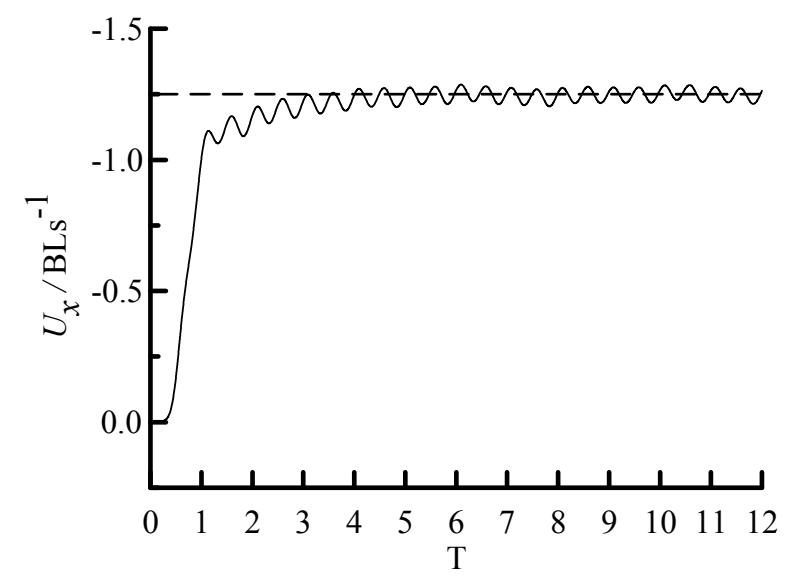

Fig. (5). Time History of Swimming Velocity over Twelve Motion Periods.

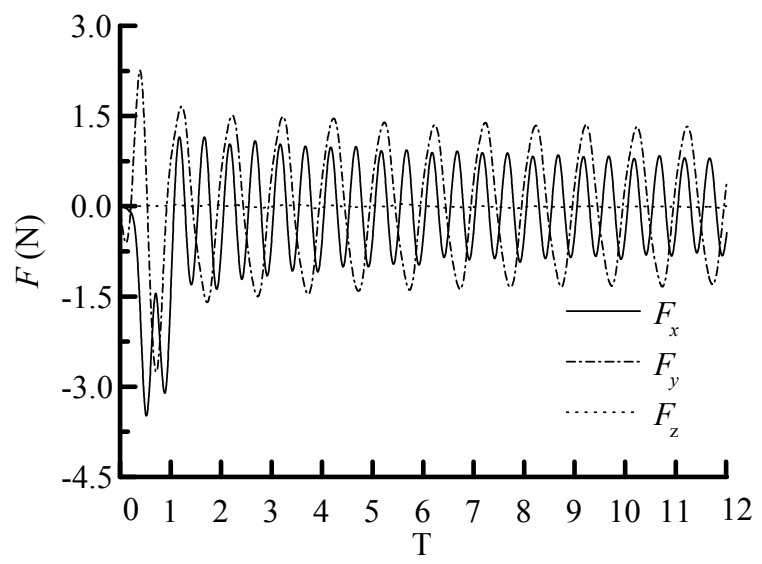

Fig. (6). Time History of Net Force, Lift Force and Lateral Force over Twelve Motion Periods.

Hz. All simulations are started from the model at rest, and then the amplitude of pectoral fin increases gradually to a designed value in rest water. The solution is considered to be converged satisfactorily when the residual value of $\mathrm{x}$ velocity is less than $1 \%$ after consecutive iterations within a time step.

The bionic model propels itself forward by undulating its paired pectoral fins in the flow field. The hydrodynamic force upon the model is calculated by integrating the pressure force and viscous force over its surface so that:

$$
\begin{aligned}
& F_{x}=\oint_{s}\left(-p n_{1}+\tau_{1 j} n_{j}\right) d s \\
& F_{y}=\oint_{s}\left(-p n_{2}+\tau_{2 j} n_{j}\right) d s \\
& F_{z}=\oint_{s}\left(-p n_{3}+\tau_{3 j} n_{j}\right) d s
\end{aligned}
$$

where $n_{i}$ is a unit normal vector pointing to the model surface, $\mathrm{s}$ is the surface of the bionic model, $\tau_{i j}$ is the viscosity stress tensor, $\bar{x}_{c}$ is the centroid coordinate value under bodyfitted coordinate system, $F_{x}$ is the net force in x direction, $F_{y}$ is the lift force in y direction and $F_{z}$ is the lateral force in $\mathrm{z}$ direction. In terms of theory, the time averaged net force
$F_{x}$, lift force $F_{y}$, lateral force $F_{z}$ is zero during steady swimming. $F_{x}$ contains two parts: thrust force $F_{T}$ and drag force $F_{D} \cdot F_{T}$ is defined as:

$F_{T}=\oint_{s}-p n_{1} d s$

Correspondingly, the non-dimensional coefficient is measured as $C_{T}=2 F_{T} / \rho S U_{T}^{2}$.

\section{RESULTS AND DISCUSSIONS}

The bionic batoid model is required to investigate the effects of biological batoid kinematics and dynamics on propulsive performance. Linear amplitude controlling equation $A_{1}$ and quadratic amplitude controlling equation $A_{2}$ are employed in this study. Pectoral fins of the model with $A_{2}$ are more flexible than that with $A_{1}$ in spanwise (aligned with yaxis).

Fig. (5) plots the time history of the velocity $U_{x}$ along xaxis direction over twelve motion periods for the bionic model, where the quadratic amplitude equation is chosen, the amplitude index $A I$ is set to 0.14 and the oscillating frequency of its paired pectoral fins is set to $2.2 \mathrm{~Hz}$. As shown in Fig. (6), the bionic model accelerates from rest to the as- 


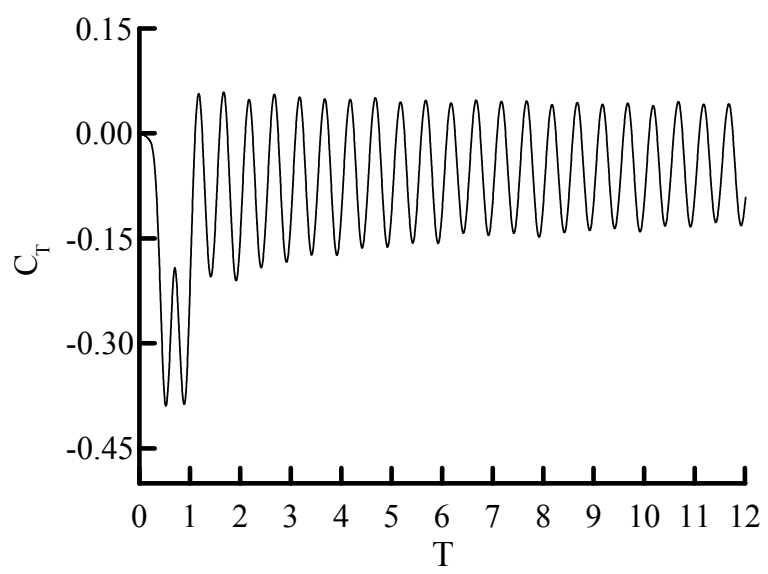

Fig. (7). Time History of Thrust Coefficient over Twelve Motion Periods.

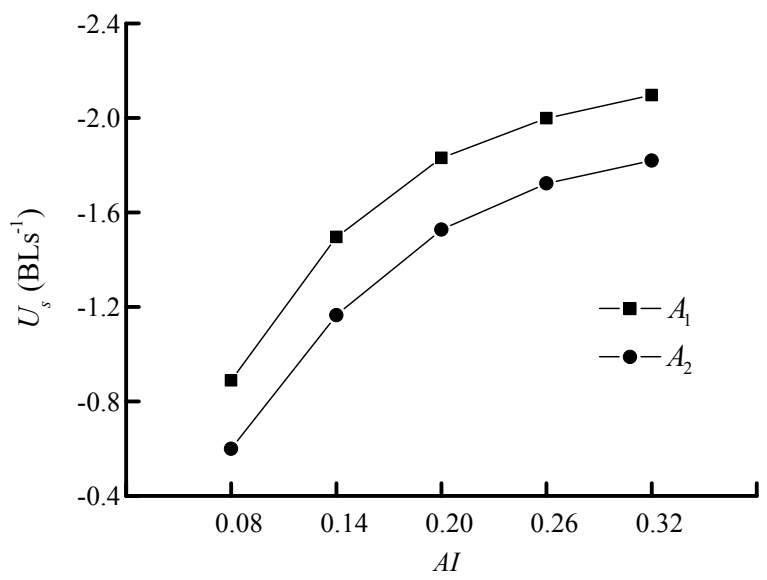

Fig. (8). Mean Swimming Velocity in x Direction versus $A I$.

ymptotic mean swimming velocity of $U_{s}=-1.25 \mathrm{BLs}^{-1}$ ( $\mathrm{BLs}^{-1}$ denotes body length per second). Since the bionic model swims aligned with the negative $\mathrm{x}$-axis, values of $U_{x}$ are all negative. Peak to peak velocity values occur twice over a period $\mathrm{T}$, the same trend occurs during the numerical simulation results in [2]. Swimming velocity $U_{x}$ oscillates with amplitude of $0.1 \mathrm{BLs}^{-1}$ during the steady-state periods, resulting in a dynamic balance.

The corresponding net force $F_{x}$, lift force $F_{y}$ and lateral force $F_{z}$ are plotted in Fig. (6). The average values of $F_{x}$, $F_{y}$ and $F_{z}$ over a steady-state period approach theoretical zero values due to the residuals of numerical calculation. The corresponding thrust coefficient $C_{T}$ is plotted in Fig. (7). Peak to peak values of thrust coefficients $C_{T}$ occur twice over a steady-state period, as the same trend of thrust coefficient has been found in experimental simulations [15, 16]. The average value of $C_{T}$ over a steady-state period is always negative, which are coincident with the value of swimming velocity $U_{x}$, meaning that the generated thrust due to the pressure force on the bionic model surface in $\mathrm{x}$-axis direction is the reason of bionic model swimming forward. Viscous drag force $F_{D}$ always impedes the locomotion of the bionic model, thus $F_{D}$ is in the opposite direction of $U_{x}$. Both $F_{T}$ and $F_{D}$ contribute to the net force $F_{x}$ which approaches to zero during steady-state swimming. In other directions, the pressure forces balance the viscous resistances, resulting in the composite forces approaching to zero.

The mean swimming velocities $U_{s}$ versus amplitude index $A I$ under linear amplitude equation $A_{1}$ and quadratic amplitude $A_{2}$ are depicted in Fig. (8), where the $A I$ is modeled at $0.08,0.14,0.20,0.26$ and 0.32 for the bionic model, which corresponds to $3.714 \mathrm{~cm}, 6.5 \mathrm{~cm}, 9.286 \mathrm{~cm}, 12.071$ $\mathrm{cm}$ and $14.857 \mathrm{~cm}$, respectively. $U_{s}$ under $A_{1}$ and $A_{2}$ increase by increasing of $A I$ while the growth rate of $U_{s}$ decrease with that. The increase rate of $U_{s}$ in $A_{1}$ is close to 


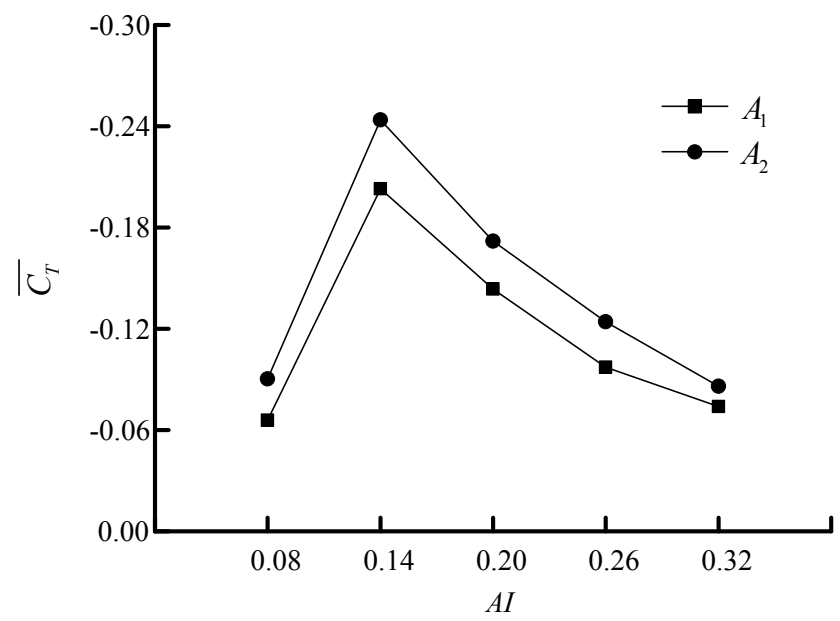

Fig. (9). Thrust Coefficient versus $A I$.

that in $A_{2}$. The simulation results of mean swimming velocities indicate that the bionic model can alter its speed by adjusting the amplitude value of the pectoral fins as the biological batoid. The higher of amplitude value, the faster of the swimming speed. Bionic model with linear amplitude controlling equation can generate higher swimming speed than that with quadratic amplitude controlling equation due to the stronger fluid-structure interactions with less spanwise flexibility.

For the simulation results shown in Fig. (9), the mean thrust coefficients $C_{T}$ over a steady-state period first increase and then decrease by increasing the amplitude index under the two amplitude controlling equations. $C_{T}$ under $A_{2}$ keep higher than that under $A_{1}$. It is remarkable that peak value emerges at $A I=0.14$ under $A_{1}$ and $A_{2}$. The mean thrust coefficients under quadratic amplitude controlling equation are always higher than that under linear amplitude controlling equation at the five amplitude indices values, meaning that better propulsive performance can be obtained from the bionic model with spanwise flexible pectoral fins. The amplitude index value with maximum mean thrust coefficient occurs at 0.14 , at which biological Raja eglanteria choose most during natural swimming. The agreement between the simulation results and the previous experimental observations shows that our numerical simulation results are acceptable.

According the previous definition of Froud efficiency, the mean net thrust is used as the numerator in calculating the Froud efficiency, thus, the net thrust is the net force $F_{x}$ which approaches to zero meaning that the Froud efficiency is zero during stead-state swimming in this paper. The results of Froud efficiency are agreed with the previous simulation results during stead-state locomotion.

\section{CONCLUSION}

A bionic batoid model is developed based on biological studies of morphologic of the natural Raja eglanteria. The model propels itself forward by undulating its paired pectoral fins from rest, which validate in its ability to create motions, forces and flows like the biological batoid and then is used to investigate the effect of pectoral fins on propulsive performance over a wide range of amplitude indices. In addition, two amplitude controlling equations $A_{1}$ and $A_{2}$ are proposed to investigate the effect of the spanwise flexibility of pectoral fins on thrust properties.

Propulsive forces and moment are created through a fluid-structure interaction between the batoid model and the fluid flow. The interaction is affected by the spanwise flexibility and the amplitude index of the pectoral fins. The mean swimming velocity increases with the increase of amplitude value. The batoid model with flexible pectoral fins generates lower swimming speed. According to the simulation results, the thrust coefficients display quite uniform variance trends as that in the experimental results, which verifies that our simulations results are acceptable. The maximum thrust coefficient emerges at the amplitude index of 0.14 , at which biological batoid swim during steady-state locomotion [4]. This study presents an effective method of altering swimming velocity and also presents a steady configuration of bionic batoid with better propulsive performance, which may be useful for future robotic works drawing inspiration from undulating rajiform swimmers.

\section{CONFLICT OF INTEREST}

The authors confirm that this article content has no conflicts of interest.

\section{ACKNOWLEDGEMENTS}

This work is supported by National Natural Science Foundation of China (NSFC 51205060) and State Key Laboratory of Robotics and System (HIT) (SKLRS 2011-ZD$03)$.

\section{REFERENCES}

[1] L. Julie and H.K. Deok, "Parameter analysis of batoid fin motions using fluid-structure interaction based simulation and design of experiments," Proceedings of the Institution of Mechanical Engineers, Part C: Journal of Mechanical Engineering Science, vol. 225, no. 8, pp. 1863-1873, 2011. 
[2] F. Gao, J. Xie and F. Yuan, "Finite-time control of nonholomic mobile robots," International Journal of Advancement in Computing Technology, vol. 4, no. 2, pp. 161-168, 2012.

[3] P. Suebsaiprom, C. Lin and S. Saimek, "Fish robot modeling and simulation: fish tail and rigid body motion", International Journal of Advancement in Computing Technology, vol. 4, no. 18, pp. 105114, 2012.

[4] L. Rosenberger, "Pectoral fin locomotion in batoid fishes: undulation versus oscillation," Journal of Experimental Biology, vol. 204, no.2, pp. 379-394, 2001.

[5] J. Tangorra, G.V. Lauder, I. W. Hunter, Rajat Mittal, Peter G.A. Madden and M. Bozkurttas, "The effect of fin ray flexural rigidity on the propulsive forces generated by a biorobotic fish pectoral fin", Journal of Experimental Biology, vol. 213, no. 23, pp. 4043-4054, 2010.

[6] E. Drucker and G. Lauder, "Function of pectoral fins in rainbow trout: behavioral repertoire and hydrodynamic forces," Journal of Experimental Biology, vol. 206, no. 5, pp. 813-826, 2003.

[7] E. Blevins and G. Lauder, "Rajiform locomotion: threedimensional kinematics of the pectoral fin surface during swimming in the freshwater stingray potamotrygon orbignyi," Journal of Experimental Biology, vol. 215, pp. 3231-3241, 2012.

[8] K. Shoele and Q. Zhu, "Numerical simulation of a pectoral fin during labriform swimming," Journal of Experimental Biology, vol. 213, no. 12, pp. 2038-2047, 2010.

[9] O. Franklin, C. Palmer and G. Dyke, "Pectoral fin morphology of batoid fishes: explaining phylogenetic variation with geometric morphometircs," Journal of Morphlolgy, vol. 275, pp. 1173-1186, 2014.

[10] W. Chen, Z. Wu, J. Liu, S. Shi and Y. Zhou, "Numerical simulation of batoid locomotion," Journal of Hydrodynamics, vol. 23, no. 5, pp. 594-600, 2011.

[11] X. Shao, D. Pan and J. Deng, "Numerical studies on the propulsion and wake structures of finite-span flapping wings with different aspect ratios," Journal of Hydrodynamic, vol. 22, no. 2, pp. 147-154, 2010.

[12] G. Matthew, K. Robert and H. Melina, "Movement and function of the pectoral fins of the larval zebrafish(Danio rerio) during slow swimming," Journal of Experimental Biology, vol. 241, no. 18, pp. 3111-3123, 2011.

[13] W. Hu, "Hydrodynamic study on a pectoral fin rowing model of a fish," Journal of Hydrodynamics, vol. 21, no.4, pp. 463-472, 2009.

[14] S. Yang, J. Qiu and X. Han, "Kinematics modeling and experiments of pectoral oscillation propulsion robotic fish," Journal of Bionic Engineering, vol. 6, no. 2, pp. 174-179, 2009.

[15] H. Melina, D. Ryan, H. Thorsen and M. Westneat, "Pectoral fin coordination and gait transitions in steadily swimming juvenile reef fishes," Journal of Experimental Biology, vol. 209, no. 19, pp. 3708-3718, 2006.

[16] Q. Liu and Z. Ji, "Optimization of propulsion performance of labriform pectoral fin," International Journal of Advancement in Computing Technology, vol. 4, no. 18, pp. 523-539, 2012.

(C) Wu et al.; Licensee Bentham Open.

This is an open access article licensed under the terms of the Creative Commons Attribution Non-Commercial License (http://creativecommons.org/licenses/by-nc/3.0/) which permits unrestricted, non-commercial use, distribution and reproduction in any medium, provided the work is properly cited. 